\title{
Comparison of two microphone array geometries for surface impedance estimation
}

\section{Richard, Antoine; Fernandez-Grande, Efren}

Published in:

Journal of the Acoustical Society of America

Link to article, DOI:

$10.1121 / 1.5116705$

Publication date:

2019

Document Version

Publisher's PDF, also known as Version of record

Link back to DTU Orbit

Citation (APA):

Richard, A., \& Fernandez-Grande, E. (2019). Comparison of two microphone array geometries for surface impedance estimation. Journal of the Acoustical Society of America, 146(1), 501-504.

https://doi.org/10.1121/1.5116705

\section{General rights}

Copyright and moral rights for the publications made accessible in the public portal are retained by the authors and/or other copyright owners and it is a condition of accessing publications that users recognise and abide by the legal requirements associated with these rights.

- Users may download and print one copy of any publication from the public portal for the purpose of private study or research.

- You may not further distribute the material or use it for any profit-making activity or commercial gain

- You may freely distribute the URL identifying the publication in the public portal

If you believe that this document breaches copyright please contact us providing details, and we will remove access to the work immediately and investigate your claim 


\section{Comparison of two microphone array geometries for surface impedance estimation}

Antoine Richard, and Efren Fernandez-Grande

Citation: The Journal of the Acoustical Society of America 146, 501 (2019); doi: 10.1121/1.5116705

View online: https://doi.org/10.1121/1.5116705

View Table of Contents: https://asa.scitation.org/toc/jas/146/1

Published by the Acoustical Society of America

\section{ARTICLES YOU MAY BE INTERESTED IN}

High-speed video microscopy and numerical modeling of bubble dynamics near a surface of urinary stone The Journal of the Acoustical Society of America 146, 516 (2019); https://doi.org/10.1121/1.5116693

Exact tuning of a vibration neutralizer for the reduction of flexural waves in beams

The Journal of the Acoustical Society of America 146, 486 (2019); https://doi.org/10.1121/1.5116690

Design of an acoustic illusion device based on a shifting medium and multi-folded transformation

The Journal of the Acoustical Society of America 146, 505 (2019); https://doi.org/10.1121/1.5118244

Estimation of surface impedance at oblique incidence based on sparse array processing

The Journal of the Acoustical Society of America 141, 4115 (2017); https://doi.org/10.1121/1.4983756

Volumetric reconstruction of acoustic energy flows in a reverberation room

The Journal of the Acoustical Society of America 145, EL203 (2019); https://doi.org/10.1121/1.5092820

Extensions to the acoustic scattering analysis for cloaks in non-uniform mean flows

The Journal of the Acoustical Society of America 146, 41 (2019); https://doi.org/10.1121/1.5115046

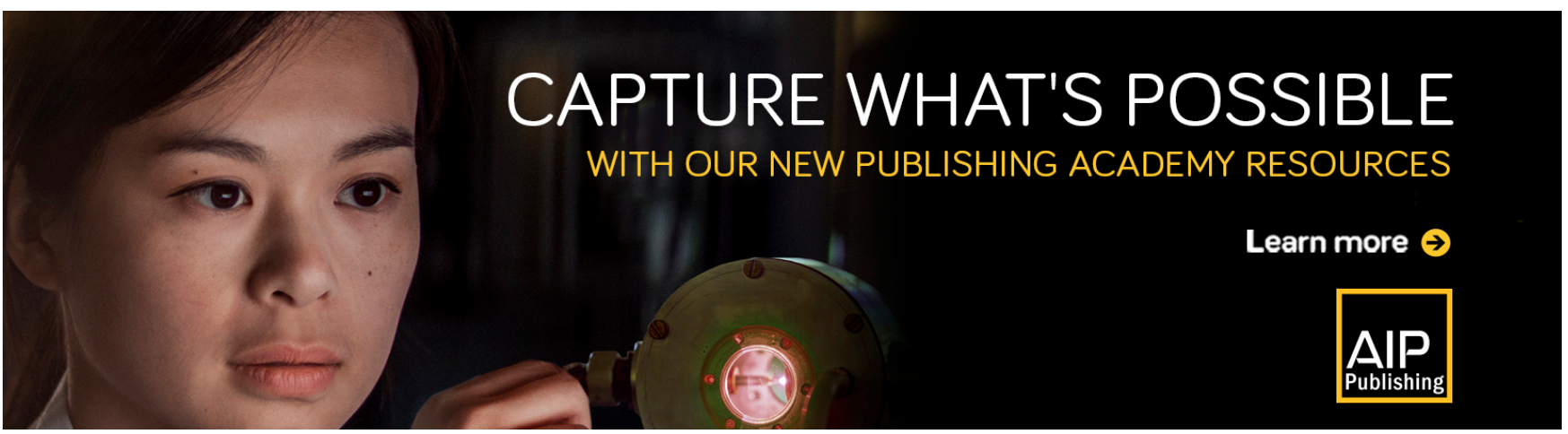




\title{
Comparison of two microphone array geometries for surface impedance estimation $(L)$
}

\author{
Antoine Richard ${ }^{\text {a) }}$ and Efren Fernandez-Grande \\ Acoustic Technology, Department of Electrical Engineering, Technical University of Denmark (DTU), \\ Building 352, DK-2800 Kongens Lyngby, Denmark
}

(Received 5 March 2019; revised 18 June 2019; accepted 26 June 2019; published online 26 July 2019)

This study examines the estimation of the surface impedance of an absorber with microphone arrays. Two array geometries are compared - a rigid spherical array and a double layer planar array. The impedance is estimated via reconstructing the sound field (pressure and particle velocity) on the absorber's surface, using a plane wave expansion. The comparison is carried out by studying the numerical properties of the two arrays as well as through experimental tests.

(C) 2019 Acoustical Society of America. https://doi.org/10.1121/1.5116705

[JDR]

Pages: 501-504

\section{INTRODUCTION}

Absorbing materials are typically characterized in terms of surface impedance $Z_{S}$ or absorption coefficient $\alpha$. However, the standardized methods to estimate these quantities only provide limited information. ${ }^{1,2}$ Therefore, alternative methods have been proposed, in particular to study the angle dependence. ${ }^{3}$ Several of these methods make use of microphone array measurements. A first approach is to separate the incident and reflected components of the sound field, using a spatial Fourier transform, ${ }^{4}$ beamforming, ${ }^{5}$ or near-field acoustic holography. ${ }^{6}$ It has also been proposed to reconstruct the sound field (pressure and particle velocity) on the tested material, from which the surface impedance is calculated. ${ }^{7-9}$

Different array configurations have been used for impedance measurements. Planar arrays are well-suited to capture the behavior of the sound field near a plane surface. ${ }^{10}$ Spherical arrays are omnidirectional sensors, that have proved useful for the experimental capture and analysis of the sound field. ${ }^{8,11}$ However, a direct comparison of such arrays seems missing in the literature. The present study investigates the influence of the array geometry on an impedance estimation method. The method is described in Ref. 8; it is based on reconstructing the sound field on the tested surface with a wave expansion model. ${ }^{12}$ Two geometries are compared in this study, namely, a planar double layer array and a rigid spherical array. The numerical properties of the wave expansion are examined, and the accuracy of the impedance estimation is investigated experimentally.

\section{METHODOLOGY}

Consider a microphone array placed close to a horizontal surface, and a given incident sound field on the surface. We assume that the measured pressures at the $M$ microphones of the array can be represented locally as a superposition of $L$ propagating plane waves, with directions of arrival distributed over the whole space. ${ }^{13}$ A matrix equation is obtained,

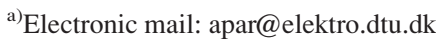

$$
\mathbf{p}=\mathbf{H} \mathbf{x}
$$

where $\mathbf{p} \in \mathbb{C}^{M}$ contains the measured pressures, $\mathbf{x} \in \mathbb{C}^{L}$ contains the unknown plane wave amplitudes, and $\mathbf{H} \in \mathbb{C}^{M \times L}$ is called the sensing matrix containing propagation functions. Usually, $L \gg M$, which makes Eq. (1) an underdetermined inverse problem. Equation (1) is solved using Tikhonov regularization, which is expressed as the optimization problem ${ }^{14}$

$$
\tilde{\mathbf{x}}=\underset{\mathbf{x}}{\operatorname{argmin}}\left(\|\mathbf{H x}-\mathbf{p}\|_{2}^{2}+\lambda\|\mathbf{x}\|_{2}^{2}\right),
$$

where $\lambda$ is a regularization parameter. $\lambda$ is selected automatically by generalized cross-validation. ${ }^{14}$ Other regularization strategies are possible, such as $\ell 1$-norm regularization, ${ }^{16}$ but the Tikhonov regularization is chosen as a conventional way of solving the inverse problem.

Once the vector $\mathbf{x}$ has been estimated, the pressure $\tilde{p}$ and the normal component of the particle velocity $\tilde{u}_{n}$ can be reconstructed on any point $\mathbf{r}_{S}$ of the surface, as a sum of plane waves. A pointwise normalized surface impedance is calculated,

$$
Z_{S}\left(\mathbf{r}_{S}\right)=-\frac{1}{\rho c} \frac{\tilde{p}\left(\mathbf{r}_{S}\right)}{\tilde{u}_{n}\left(\mathbf{r}_{S}\right)} .
$$

The pointwise impedance is then averaged over a small surface in order to smooth out variance errors due to noise. Finally, an absorption coefficient is estimated using a plane wave model,

$$
\alpha=1-\left|\frac{\tilde{Z}_{S} \cos (\psi)-1}{\tilde{Z}_{S} \cos (\psi)+1}\right|^{2},
$$

where $\tilde{Z}_{S}$ is the spatially averaged surface impedance and $\psi$ is the angle of incidence of the incident wave. This approximation is valid if the incident wave is quasi-plane, which we assume to be true if the sound source is sufficiently far away from the surface.

\section{COMPARISON OF MICROPHONE ARRAYS}

We consider an experimental setup that consists of a large plane sample placed on a backing plate, an omnidirectional 


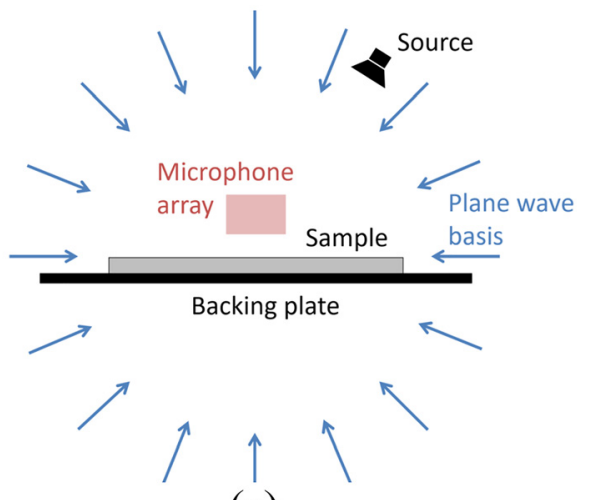

(a)

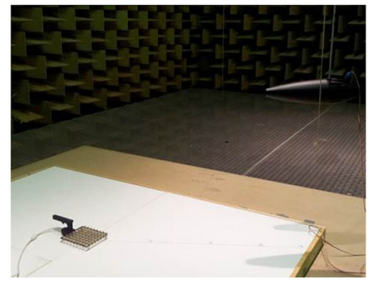

(b)

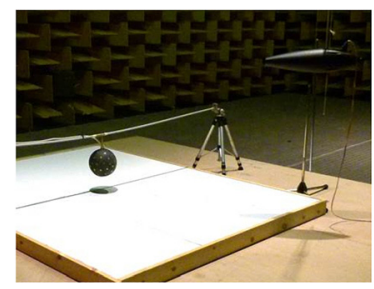

(c)
FIG. 1. (Color online) Experimental setup. (a) Sketch. (b) Planar array setup. (c) Spherical array setup.

source, and a microphone array close to the surface of the sample, as shown in Fig. 1(a). The setup is placed in an anechoic room of about $1000 \mathrm{~m}^{3}$ at the Technological University of Denmark. Two different array geometries are tested, a planar double layer array and a rigid spherical array. A basis of $L=256$ plane waves is used for the plane wave expansion. The directions of arrival of the plane waves are uniformly distributed over a sphere.

The planar array [Fig. 1(b)] consists of $M_{1}=128$ channels, arranged in two square layers of 64 microphones, with a vertical spacing of $2.9 \mathrm{~cm}$ between the two layers and a horizontal spacing of $2.5 \mathrm{~cm}$. The array aperture is $17.5 \mathrm{~cm} \times 17.5 \mathrm{~cm}$. We assume that the planar array does not disturb the sound field. Therefore, the sensing matrix coefficients correspond to plane wave terms in free-field,

$$
\mathbf{H}_{i, j}=e^{-\mathrm{j} \mathbf{k}_{j} \cdot \mathbf{r}_{i}},
$$

where $\mathbf{k}_{j}$ is the wave vector of the $j$ th plane wave $(j=1, \ldots$, $L)$ and $\mathbf{r}_{i}$ is the position vector of the $i$ th microphone $\left(i=1, \ldots, M_{1}\right)$.

The spherical array [Fig. 1(c)] contains $M_{2}=64$ uniformly distributed microphones, which are flush-mounted on a rigid sphere of radius $a=9.75 \mathrm{~cm}$. The average spacing between the microphones is about $4 \mathrm{~cm}$. The spherical array introduces scattering, which is accounted for analytically in the sensing matrix, ${ }^{15}$

$$
\mathbf{H}_{i, j}=-\frac{4 \pi}{(k a)^{2}} \sum_{n=0}^{\infty} \sum_{m=-n}^{n} \frac{\mathrm{j}^{n+1}}{h_{n}^{\prime(2)}(k a)} Y_{n}^{m}\left(\theta_{i}, \varphi_{i}\right) Y_{n}^{m *}\left(\theta_{0, j}, \varphi_{0, j}\right),
$$

where $k$ is the wavenumber, $\left(a, \theta_{i}, \varphi_{i}\right)$ are the spherical coordinates of the $i$ th microphone on the sphere $\left(i=1, \ldots, M_{2}\right)$, $\left(\theta_{0, j}, \varphi_{0, j}\right)$ represents the direction of arrival of the $j$ th plane wave $(j=1, \ldots, L), h_{n}^{(2)}$ is the spherical Hankel function of the second kind of order $n$ and $Y_{n}^{m}$ is the spherical harmonic of order $(n, m)$.

First, the numerical differences between Eq. (5) and Eq. (6) are examined. It is known that redundancies between the columns of $\mathbf{H}$ introduce linear dependencies that make it rank-deficient. In that case, the inversion of Eq. (1) becomes more sensitive to noise. ${ }^{16}$ These redundancies are studied using the Gram matrix $\Gamma \in \mathbb{R}^{L \times L}$, which contains the correlations between the columns of $\mathbf{H}$, noted $\mathbf{h}_{1}, \ldots, \mathbf{h}_{L}$. The term $\left(i^{\prime}, j^{\prime}\right)$ of $\boldsymbol{\Gamma}$ is given by ${ }^{16}$

$$
\Gamma_{i^{\prime}, j^{\prime}}=\frac{\left|\mathbf{h}_{i^{\prime}}^{*} \mathbf{h}_{j^{\prime}}\right|}{\left\|\mathbf{h}_{i^{\prime}}\right\|\left\|\mathbf{h}_{j^{\prime}}\right\|},
$$

with $i^{\prime}=1, \ldots, L$ and $j^{\prime}=1, \ldots, L . \Gamma$ is a symmetric matrix, with terms ranging from 0 to 1 . Large values in $\Gamma$ indicate pairs of plane waves that have a similar effect on the array, so they cannot be easily distinguished.

Figures 2(a)-2(f) represent the elements of the Gram matrix for the two array configurations at 250, 1000, and $4000 \mathrm{~Hz}$. In general, plane waves with close directions of arrival are highly correlated, which explains for instance the larger coefficients close to the diagonal of $\Gamma$. At $250 \mathrm{~Hz}$ [Figs.

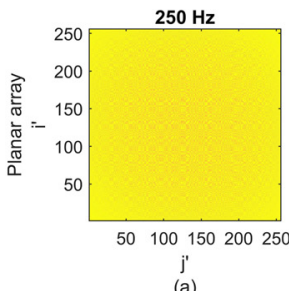

(a)

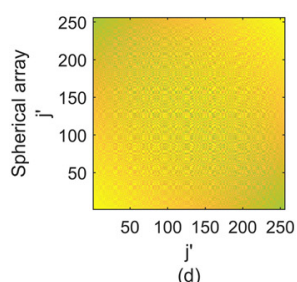

(d)

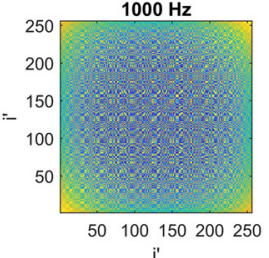

(b)

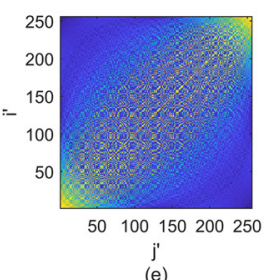

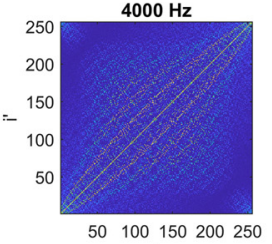

j'

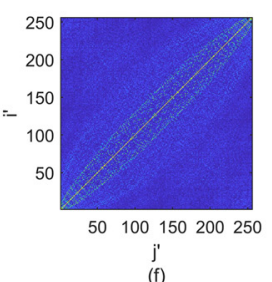

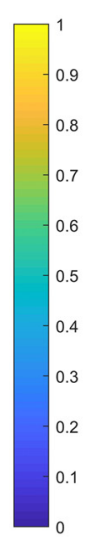

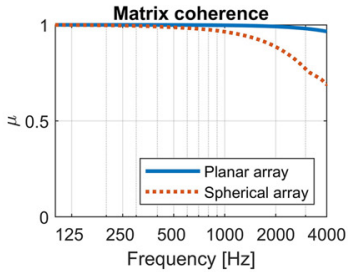

(g)

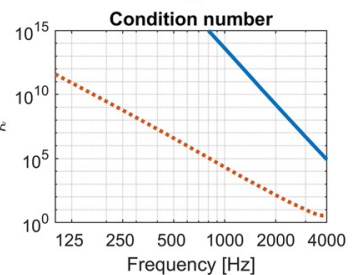

(h)

FIG. 2. (Color online) Gram matrix coefficients for the planar array at (a) $250 \mathrm{~Hz}$, (b) $1000 \mathrm{~Hz}$, and (c) $4000 \mathrm{~Hz}$. Gram matrix coefficients for the spherical array at (d) $250 \mathrm{~Hz}$, (e) $1000 \mathrm{~Hz}$, and (f) $4000 \mathrm{~Hz}$. (g) Matrix coherence. (h) Condition number. 
2(a) and 2(d)], both microphone arrays show large values in $\boldsymbol{\Gamma}$, as the wavelength $(1.4 \mathrm{~m})$ is much larger than the two arrays' dimensions. The correlations for the planar array in Fig. 2(a) are generally higher than for the spherical array in Fig. 2(d), due to the smaller distances between its microphones. In addition, values in the upper left corner (small $i^{\prime}$ and large $j^{\prime}$ ) are higher for the planar array. These elements correspond to correlations between plane waves traveling normally to the layers of the planar array. Such plane waves would yield the same pressure on an entire layer, which introduces redundancies in the sensing matrix. At $1000 \mathrm{~Hz}$ [Figs. 2(b) and 2(e)], the values of $\Gamma$ are lower than at $250 \mathrm{~Hz}$ for both arrays, due to the smaller wavelength $(34 \mathrm{~cm})$. The differences observed in the upper left corner are also more pronounced. At $4000 \mathrm{~Hz}$ [Figs. 2(c) and 2(f)], the correlations continue to decrease for both arrays, with still higher values for the planar array. Figure 2(g) presents the matrix coherence $\mu$, which is the maximum off-diagonal of the Gram matrix, as a function of frequency. $\mu$ decreases with frequency and it is overall higher for the planar array. Figure 2(h) shows the condition number of $\mathbf{H}$, denoted $\kappa$, which is the ratio between the maximum and the minimum singular value of $\mathbf{H} . \kappa$ is lower for the spherical array, which indicates that the inverse problem Eq. (1) is better conditioned for the spherical array. ${ }^{14}$ This is also a consequence of the lower coherence, as $\kappa$ is bounded by a function of $\mu{ }^{16}$ The sensing matrices remain severely ill-conditioned at low frequencies for both configurations, where the same information is measured by all the microphones. In conclusion, the spherical array captures less redundant information on the sound field than the planar array, leading to a better conditioned inverse problem.

\section{EXPERIMENTAL IMPEDANCE ESTIMATION}

The surface impedance of a porous absorber sample is estimated with both arrays. The sample is a plate of glass wool. It has a flow resistivity of $10900 \mathrm{Nsm}^{-4}$ and dimensions of $1.8 \mathrm{~m} \times 2.4 \mathrm{~m} \times 10 \mathrm{~cm}$. The source is placed at a distance of $1.5 \mathrm{~m}$ from the sample. The planar array is placed directly on the sample, its layers parallel to the surface, with a distance of $1.3 \mathrm{~cm}$ between the lower layer and the surface [see Fig. 1(b)]. The spherical array is suspended above the sample. Due to experimental constraints, the distance between the surface and the bottom of the sphere is $10 \mathrm{~cm}$ [see Fig. 1(c)]. The estimated surface impedance is an average of 441 pointwise impedances calculated on a grid of dimensions $10 \times 10 \mathrm{~cm}^{2}$ below the microphone arrays.

Figure 3 shows the estimated surface impedance and absorption coefficient for the two arrays, at $0^{\circ}, 30^{\circ}$, and $45^{\circ}$ incidence. A theoretical surface impedance is obtained as $Z_{S}(\psi)$ $=-\mathrm{j} Z_{C}\left(k / k_{n}\right) \cot \left(k_{n} h\right)$, where $k_{n}=\sqrt{-\gamma^{2}-k^{2} \sin ^{2}(\psi)}, h$ is the sample's thickness, and the parameters $Z_{C}$ and $\gamma$ are calculated using Miki's model. ${ }^{17,18}$ An absorption curve from an impedance tube measurement is also used as reference for normal incidence in Fig. 3(d) (tube diameter $3 \mathrm{~cm}$ ). At $0^{\circ}$ incidence [Figs. 3(a) and 3(d)], the spherical array performs better than the planar array below $400 \mathrm{~Hz}$, due to its better numerical conditioning, discussed in Sec. III. Between 400 and $1000 \mathrm{~Hz}$, the estimation is accurate with both microphone arrays. Above $1000 \mathrm{~Hz}$, the planar array results are more accurate than the spherical array ones. Indeed, the planar array benefits from being very close to the reconstruction surface, so that errors in the wave expansion are not amplified in the reconstruction. ${ }^{12}$ The estimation with the spherical array can be considerably improved using other regularization techniques to solve Eq. (1) (e.g., compressive sensing) ${ }^{8,16}$ However, compressive sensing is not suitable for the planar array, due to the high coherence of its sensing matrix observed in Fig. 2(g). At $30^{\circ}$ incidence, in Figs. 3(b) and $3(\mathrm{e})$, the impedance estimates are similar to those found at normal incidence, which shows that the surface impedance of this particular sample can be considered independent of the angle up to $30^{\circ}$. At $45^{\circ}$ incidence [Figs. 3(c) and 3(f)], the spherical array result shows deviations from the theoretical value, due to stronger edge diffraction and a smaller normal particle velocity, which lead to reconstruction errors. Overall, although the numerical

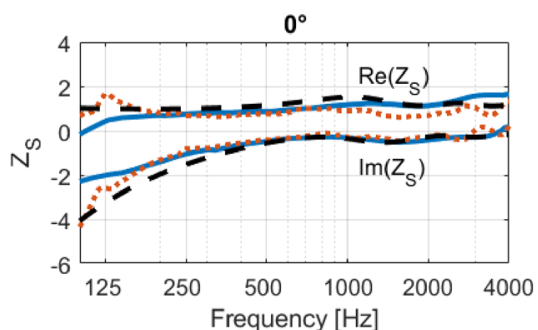

(a)

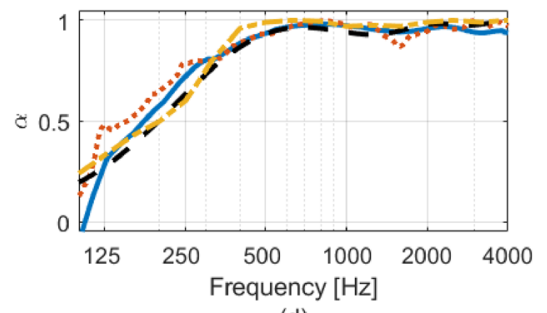

(d)

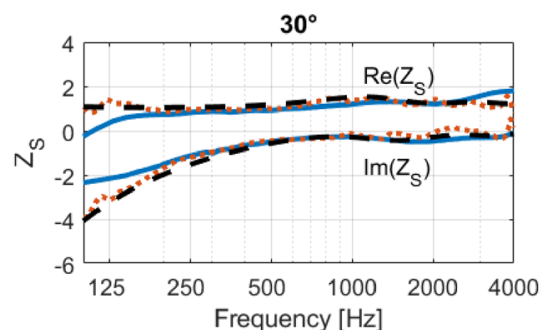

(b)

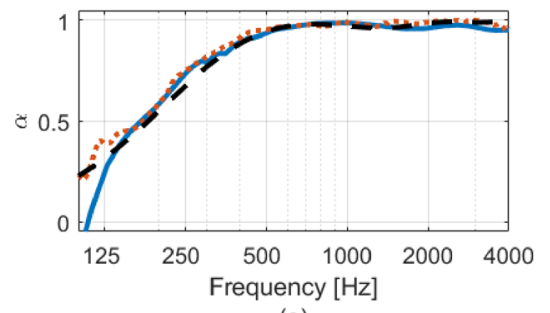

(e)

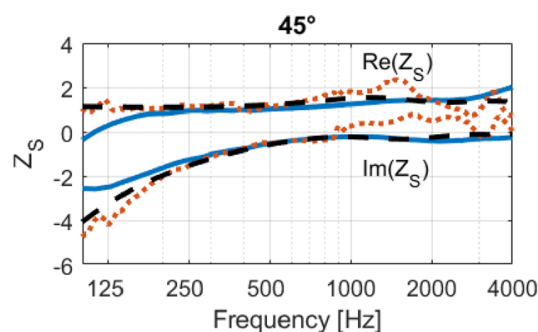

(c)

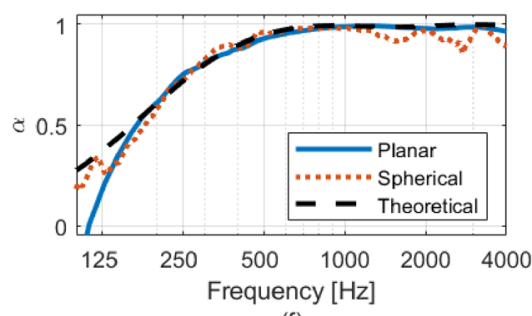

(f)

FIG. 3. (Color online) Estimated surface impedance at (a) $0^{\circ}$, (b) $30^{\circ}$, and (c) $45^{\circ}$ incidence. Estimated absorption coefficient at (d) $0^{\circ}$, (e) $30^{\circ}$, and (f) $45^{\circ}$ incidence. Planar array (-), spherical array ( . .), theoretical results (- - ), impedance tube measurement (- - -). 
conditioning of the spherical array does lead to a better impedance estimation at low frequencies, the small distance between the sample and the planar array $(1.3 \mathrm{~cm})$ is beneficial at high frequencies and larger incidence angles. The estimation with the planar array worsens as the distance increases (not shown).

\section{CONCLUSION}

This study presents a comparison of two microphone arrays (double layer planar array and spherical array with a regular spacing) for estimating the impedance of a surface, based on a plane wave expansion. In this specific study, the spherical array captures less redundant information on the sound field, which improves the numerical conditioning of the wave expansion. However, the sound field is more accurately reconstructed with the planar array at high frequencies, due to its proximity to the sample. Although the results are specific to the present study, they demonstrate that the choice of a suitable array depends on several aspects, including numerical properties due to the array configuration (size, microphone spacing, etc.) as well as experimental conditions like the distance to the sample.

\section{ACKNOWLEDGMENTS}

The authors would like to thank Jonas Brunskog and Cheol-Ho Jeong for helpful discussions, Jørgen Hald, Karim Haddad, and Wookeun Song (Brüel \& Kjær) for the experimental planar array data, and Mélanie Nolan for the impedance tube measurement data. The measurements were part of the Danish Sound Innovation Network project called in situ acoustic impedance estimation based on sparse array processing, in collaboration with Brüel \& Kjær, Bang \& Olufsen, Ecophon, and Odeon.

${ }^{1}$ ISO 10534-2, "Acoustics—Determination of sound absorption coefficient and impedance in impedance tubes" (International Organization for Standardization, Geneva, Switzerland, 1998).
${ }^{2}$ ISO 354, "Acoustics-Measurement of sound absorption in a reverberation room" (International Organization for Standardization, Geneva, Switzerland, 2003).

${ }^{3}$ E. Brandão, A. Lenzi, and S. Paul, "A review of the in situ impedance and sound absorption measurement techniques," Acta Acust. Acust. 101, 443-463 (2015).

${ }^{4}$ M. Tamura, "Spatial Fourier transform method of measuring reflection coefficients at oblique incidence. I. Theory and numerical examples," J. Acoust. Soc. Am. 88(5), 2259-2264 (1990).

${ }^{5}$ J. Rathsam and B. Rafaely, "Analysis of in situ acoustic absorption using a spherical microphone array," Appl. Acoust. 89, 273-280 (2015).

${ }^{6}$ M. Ottink, J. Brunskog, C.-H. Jeong, E. Fernandez-Grande, P. Trojgaard, and E. Tiana-Roig, "In situ measurements of the oblique incidence sound absorption coefficient for finite sized absorbers," J. Acoust. Soc. Am. 139(1), 41-52 (2016).

${ }^{7}$ Y.-B. Zhang, W.-L. Lin, and C.-X. Bi, "A technique based on the equivalent source method for measuring the surface impedance and reflection coefficient of a locally reacting material," in Proceedings of Internoise, Melbourne (2014).

${ }^{8}$ A. Richard, E. Fernandez-Grande, J. Brunskog, and C.-H. Jeong, "Estimation of surface impedance at oblique incidence based on sparse array processing," J. Acoust. Soc. Am. 141(6), 4115-4125 (2017).

${ }^{9}$ J. Hald, W. Song, K. Haddad, C.-H. Jeong, and A. Richard, "In-situ impedance and absorption coefficient estimations using a double-layer microphone array," Appl. Acoust. 143, 74-83 (2019).

${ }^{10} \mathrm{~S}$. Gade, J. Gomes, and J. Hald, "Use of hand held array for NVH measurement in the automotive industry," in Internoise, Innsbruck (2013).

${ }^{11}$ B. Rafaely, "Analysis and design of spherical microphone arrays," IEEE Trans. Speech Audio Process. 13(1), 135-143 (2005).

${ }^{12}$ E. Fernandez-Grande, "Sound field reconstruction using a spherical microphone array," J. Acoust. Soc. Am. 139(3), 1168-1178 (2016).

${ }^{13}$ E. G. Williams, Sound Radiation and Nearfield Acoustical Holography (Academic, San Diego, 1999), Chap. 2.

${ }^{14}$ P. C. Hansen, Rank-Deficient and Discrete Ill-Posed Problems. Numerical Aspects of Linear Inversion (SIAM, Philadelphia, PA, 1998).

${ }^{15} \mathrm{~B}$. Rafaely, "Plane wave decomposition of the sound field on a sphere by spherical convolution," J. Acoust. Soc. Am. 116, 2149-2157 (2004).

${ }^{16}$ E. Fernandez-Grande, A. Xenaki, and P. Gerstoft, "A sparse equivalent source method for near-field acoustic holography," J. Acoust. Soc. Am. 141(1), 532-542 (2017).

${ }^{17}$ J. F. Allard and N. Atalla, Propagation of Sound in Porous Media: Modelling Sound Absorbing Materials, 2nd ed. (Wiley, Chichester, UK, 2009), Chap. 3, pp. 29-43.

${ }^{18}$ Y. Miki, "Acoustical properties of porous materials-Modifications of Delany-Bazley models,” J. Acoust. Soc. Am. 11, 19-24 (1990). 